\title{
Allogeneic stem-cell transplantation-resolving questions of timing, remission and the individual
}

$\mathrm{P}$

atients with acute myeloid leukaemia (AML) have a number of options available to them for treatment. These include, but are not limited to, chemotherapy and stem-cell transplantation and, if the patient relapses, reinduction chemotherapy. Further complicating matters, patients with AML are typically categorized according to their cytogenetic features. Patients with 'favourable risk' cytogenetics tend to do better with chemotherapy-based treatments than those with 'unfavourable risk' cytogenetics. Despite a wealth of treatment strategies in our armamentarium, important questions remain as to which patients benefit most from stem-cell transplantation and when in the history of the disease the most benefit can be gained from transplantation. A new study published in the Journal of Clinical Oncology quantifies the benefits of salvage treatments in those who did not initially undergo stem-cell transplantation in their first complete remission (CR1) and assesses the benefits of leaving transplantation to the second complete remission (CR2).

"One of the issues that comes into play when advising a patient with AML in first remission about going to transplant is not only the chances of the transplant being effective, but also the chance they will relapse if they don't have a transplant," explains lead investigator Alan Burnett. The overarching goal of the study was to leverage clinical trial data to determine whether there is any benefit in delaying stem-cell transplantation to CR2. Should a transplant be given to all patients in CR1? Should transplant only be reserved for those who relapsed after first-line chemotherapy? From a health policy perspective, the costs involved in expansive use of transplantation could be

Original article Burnett, A. K. et al. Curability of patients with acute myeloid leukemia who did not undergo transplantation in first remission. J. Clin. Oncol. doi:10.1200/JC0.2011.40.5977 significant, especially if a considerable number of patients don't stand to benefit.

Burnett's team looked at patients who were enrolled in several AML trials in the UK (specifically, the Medical Research Council AML10, AML12 and AML15 trials), who did not receive a transplant in CR1 and went on to relapse, which amounted to 1,271 patients out of a total of 3,919. Of these, 642 entered CR2, 433 of whom received a stem-cell transplant. Importantly, this study included patients in all three cytogenetic risk groups who had received transplants from sibling or matched nonsibling donors, autografts or reduced-intensity transplantsencompassing a whole range of transplant options. "No previous study had this number of patients to precisely measure the impact that a transplant can make if given after relapse," comments Burnett.

The data showed that $19 \%$ of all patients who did not undergo a transplant in CR1 were alive at 5 years. By comparison, the overall survival after stem-cell transplant (regardless of technique) in CR2 was 2.8 years; that these patients are less likely to survive is intuitive. Highpowered statistical methods (MantelByar comparisons) were used to control for selection factors, which-given the heterogeneity of the patients in the study in terms of disease and treatment histories-were likely to influence the results. The survival in this case was $44 \%$ with a median follow-up duration of 5.4 years, which was better than the $21 \%$ survival rate of those who did not receive a transplant. Taking into account all the transplant options as well as the prognostic influence of white blood cell count, duration of the first remission and the cell source used in the transplant, the researchers found that only the duration of CR1 was associated with the outcome.

Using landmark analysis starting at the median time to transplant in CR1 (127 days in this patient group), the researchers found a survival rate of
$72 \%$ for those who did not undergo a transplant in CR1 and were in the cytogenetic favourable-risk group, which suggests that these patients would not benefit from stem-cell transplant at any point during their disease. "I was a little surprised that for good-risk patients, who are not normally transplanted in CR1 anyway, the result from chemotherapy alone after relapse was not different from a transplant after relapse," says Burnett.

By contrast, the landmark survival of intermediate-risk patients was considerably lower (approximately 50\%), which suggests these patients can benefit from transplantation. However, the data show that transplantation can be delayed until after relapse, which would necessitate fewer transplants and save patients from considerable burden caused by the treatment. Burnett continues: "our data suggests that, in some risk groups, cure can be possible for the same number of patients with a lot fewer transplants given."

Although this work sheds much-needed light on the subtleties of AML treatment, increasingly accurate methods are needed to identify patients who require stem-cell transplantation in the first-line. Future clinical trials that assess patients in a headto-head manner will also clarify the role of transplantation in CR2.

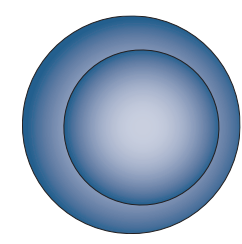

Mina Razzak

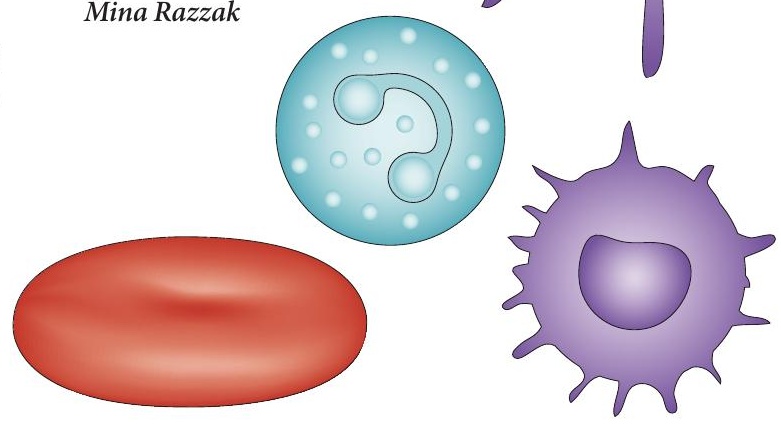

